\title{
Surface Harmonic Expansions of Products of Cartesian Coordinates
}

\author{
By A. B. Otis, Jr. and M. P. Barnett*
}

1. Introduction. Products of Cartesian coordinates must be expressed in terms of zonal and tesseral harmonics, and vice versa, in the analysis of physical problems of several types. If $(x, y, z)$ are the coordinates of a point, expressed in some Cartesian coordinate system, and $(r, \theta, \phi)$ are the coordinates of the same point expressed in the corresponding polar coordinate system, then

$$
x / r=\sin \theta \cos \phi, \quad y / r=\sin \theta \sin \phi, \quad z / r=\cos \theta .
$$

We use Hobson's definition of the associated Legendre functions [1].

Any product of non-negative powers of $(x / r),(y / r)$, and $(z / r)$ can be expanded as a linear combination of harmonics $P_{n}(\cos \theta), P_{n}{ }^{m}(\cos \theta) \cos m \phi$ and $P_{n}{ }^{m}(\cos \theta) \sin m \phi$ in which $n$ and $m$ take appropriate values. This expansion is given formally by Equation (2), which defines the coefficients $a_{u, v, w}^{n, m}$. We consider only non-negative integer values of $u, v, w$.

$$
x^{u} y^{v} z^{w} / r^{u+v+w}=\sum_{n, m} a_{u, v, w}^{n, m} P_{n}^{m}(\cos \theta) \underset{\sin }{\cos } m \phi .
$$

In this equation, terms in $\cos m \phi$ are used when $v$ is even, and terms in $\sin m \phi$ when $v$ is odd. The coefficients are zero unless

$$
\begin{gathered}
(u \bmod 2+v \bmod 2+w \bmod 2) \leqq n \leqq u+v+w, \\
\quad(u \bmod 2+v \bmod 2) \leqq m \leqq u+v, \\
u+v+w+n \text { even, } u+v+m \text { even. }
\end{gathered}
$$

It is convenient to define $a_{u, v, w}^{n, m}$ to be zero for negative $n, m$, and for $m>n$.

We use the inverse transformation that is given formally by Equation (4). This equation, together with the constraint

$$
u+v=m
$$

defines the coefficients $b_{u, v, w}^{n, m}$, for $0 \leqq m \leqq n$.

$$
P_{n}^{m}(\cos \theta) \underset{\sin }{\cos } m \phi=\sum_{u, v, w} b_{u, v, w}^{n, m}(x / r)^{u}(y / r)^{v}(z / r)^{w} .
$$

The coefficients are zero unless

$$
\begin{aligned}
& (n-m) \bmod 2 \leqq w \leqq n-m, \quad 0 \leqq u, v \leqq m, \\
& u+v+w+n \text { even, } \\
& v \text { is even when } \cos m \phi \text { occurs on the left-hand side of (4), } \\
& v \text { is odd when } \sin m \phi \text { occurs on the left-hand side of (4). }
\end{aligned}
$$

Received July 16, 1963. Revised January 9, 1964.

* Present address: Institute of Computer Science, University of London, England. 
The coefficients in the expansions (2) and (4) are of use in the applications of quantum theory to chemical and other problems [2]. Some tables of these coefficients have been constructed by a digital computer by use of some simple recurrence formulae. The results are expressed as rational fractions. They have been typeset photographically from the computer output in a mechanical fashion that avoided manual keyboard operations. The tables for $0 \leqq u+v+w \leqq 9$ that were produced in this way are given in [3]. Excerpts are reproduced here as Tables 1 and 2 of this note. The computer programs can be used to generate the coefficients for higher values of the indices if necessary.

2. Recurrence Formulae for $a_{u, v, w}^{n, m}$ Coefficients. The well-known recurrence formulae for the Legendre functions can be used to derive recurrence formulae for the $a_{u, v, w}^{n, m}$ coefficients. The formula to raise $w$ is obtained by constructing two series for $(x / r)^{u}(y / r)^{v}(z / r)^{w+1}$ from the right-hand side of Equation (2) and equating terms. To obtain the first series, write $w+1$ in place of $w$. To obtain the second series, write $n^{\prime}$ in place of the summation index $n$, and multiply the summand by $\cos \theta$. This gives

$$
\sum_{n, m} a_{u, v, w+1}^{n, m} P_{n}^{m}(\cos \theta) \underset{\sin }{\cos } m \phi=\sum_{n^{\prime}, m} a_{u, v, w}^{n^{\prime}, m} \cos \theta P_{n^{\prime}}^{m}(\cos \theta) \underset{\sin }{\cos } m \phi .
$$

Replace the product $\cos \theta P_{n^{\prime}}^{m}(\cos \theta)$ in the summand on the right-hand side of Equation (6) by terms in $P_{n^{\prime} \pm 1}^{m}(\cos \theta)$. Substitute $n=n^{\prime} \pm 1$ in the resulting terms in $P_{n^{\prime} \pm 1}^{m}(\cos \theta)$. Rearrange the summation on the right-hand side so that $n$ is the index of summation. Equate coefficients of $P_{n}^{m}(\cos \theta)$. This gives

$$
a_{u, v, w+1}^{n, m}=\frac{n-m}{2 n-1} a_{u, v, w}^{n-1, m}+\frac{n+m+1}{2 n+3} a_{u, v, w}^{n+1, m} .
$$

It can be shown that this derivation is valid in the cases that $n=0$ and $n=m$, at which times one or other of the terms on the right-hand side of Equation (7) is zero in accordance with the convention that is stated after the restrictions (3).

The formula to raise $u$ is obtained in a similar manner, by constructing two series for $(x / r)^{u+1}(y / r)^{v}(z / r)^{w}$ from the right-hand side of Equation (2), and equating terms. To obtain the first series, write $u+1$ in place of $u$. To obtain the second series, write $n^{\prime}$ in place of $n$, and $m^{\prime}$ in place of $m$, and multiply the summand by $\sin \theta \cos \phi$. Use the recurrence formulae for $\sin \theta P_{n^{\prime}}^{m^{\prime}}(\cos \theta)$, and the elementary properties of the circular functions of $\phi$, to replace

$$
\sin \theta P_{n^{\prime}}^{m^{\prime}}(\cos \theta) \cos \phi \underset{\sin }{\cos } m^{\prime} \phi \text { by terms in } P_{n^{\prime}{ }^{\prime} \pm 1}^{\prime^{\prime} \pm 1}(\cos \theta) \underset{\sin }{\cos }\left\{\left(m^{\prime} \pm 1\right) \phi\right\} .
$$

The four terms in $\left(n^{\prime}+1, m^{\prime}+1\right),\left(n^{\prime}-1, m^{\prime}+1\right),\left(n^{\prime}+1, m^{\prime}-1\right)$ and $\left(n^{\prime}-1, m^{\prime}-1\right)$ result from this process. Substitute $n=n^{\prime}+1$ in the terms in $\left(n^{\prime}+1, m^{\prime} \pm 1\right)$. Substitute $n=n^{\prime}-1$ in the terms in $\left(n^{\prime}-1, m^{\prime} \pm 1\right)$. Substitute $m=m^{\prime}+1$ in the terms in $\left(n^{\prime} \pm 1, m^{\prime}+1\right)$. Substitute $m=m^{\prime}-1$ in the terms in $\left(n^{\prime} \pm 1, m^{\prime}-1\right)$. Rearrange the summation so that $n$ and $m$ are the indices of summation. Equate coefficients in

$$
P_{n}^{m}(\cos \theta) \underset{\sin }{\cos } m \phi .
$$


This gives

$$
\begin{aligned}
a_{u+1, v, w}^{n, m}= & \frac{1}{2}\left[\frac{1}{(2 n+3)}\left\{\left(1+\delta_{m, 1}\right) a_{u, v, w}^{n+1, m-1}-(n+m+1)(n+m+2) a_{u, v, w}^{n+1, m+1}\right\}\right. \\
& \left.-\frac{1}{(2 n-1)}\left\{\left(1+\delta_{m, 1}\right) a_{u, v, w}^{n-1, m-1}-(n-m)(n-m-1) a_{u, v, w}^{n-1, m+1}\right\}\right]
\end{aligned}
$$

where

$$
\delta_{1,1}=1 ; \quad \delta_{m, 1}=0, m \neq 1,
$$

in the Kronecker delta notation. The derivation of the recurrence formula (10) that is used to raise the value of $v$ is completely analogous to the derivation of Equation $(8)$, with $(y / r)$ playing the part that $(x / r)$ played for $(8)$.

$$
\begin{aligned}
& a_{u, v+1, w}^{n, m}=\frac{1}{2}(-1)^{v}\left[\frac { 1 } { ( 2 n + 3 ) } \left\{\left(1+\delta_{m, 1}\right) a_{u, v, w}^{n+1, m-1}\right.\right. \\
&\left.+(n+m+1)(n+m+2) a_{u, v, w}^{n+1, m+1}\right\}-\frac{1}{(2 n-1)}\left\{\left(1+\delta_{m, 1}\right) a_{u, v, w}^{n-1, m-1}\right. \\
&\left.\left.+(n-m)(n-m-1) a_{u, v, w}^{n-1, m+1}\right\}\right] .
\end{aligned}
$$

3. Mechanization of Recurrence Procedure. The order in which the $a_{u, v, w}^{n, m}$ coefficients are constructed conveniently can be described in terms of the index $s$ that is defined by

$$
s=u+v+w .
$$

In terms of this index, the nonzero $a_{u, v, w}^{n, m}$ coefficients can be constructed in the order (12) for any positive integer $s_{\max }$.

$$
\begin{gathered}
s=0(1) s_{\max }, \quad u=0(1) s, \quad v=0(1) s-u, \quad w=s-u-v, \\
n=(u \bmod 2+v \bmod 2+w \bmod 2)(2) s \\
m=(u \bmod 2+v \bmod 2)(2)(u+v) .
\end{gathered}
$$

The process is started by use of Equation (13)

$$
a_{0,0,0}^{0,0}=1 \text {. }
$$

Each $a_{u, v, w}^{n, m}$ coefficient for $s \geqq 1$ is computed by Equation (8) if $w=v=0$, by Equation (10) if $w=0, v \neq 0$, and by Equation (7) if $w \neq 0$.

To mechanize this recurrence scheme, the coefficients must be stored, as they are formed, in a way that allows the program to retrieve the $a_{u, v, w}^{n, m}$ coefficient of any $u, v, w, n, m$ values which it requires for further use in an application of a recurrence formula. This type of issue arises in the mechanization of any recurrence scheme that generates quantities which depend on several indices, and it can be regarded as a mathematical consideration which is as independent of computer models and programming languages as the analysis of round off errors or the derivation of the recurrence formulae themselves. A formalism to deal with this matter has been reported recently [4]. In this formalism, the list of $a_{u, v, w}^{n, m}$ coefficients arranged in the 
order (12) is denoted by

$$
\bigwedge_{s=0}^{s_{\max }} \bigwedge_{u=0}^{s} \bigwedge_{v=0}^{s-u} \bigwedge_{\nu=0}^{\nu_{\max }} \bigwedge_{\mu=0}^{\mu_{\max }} a_{u, v, s-u-v}^{n, m}
$$

where

$$
\begin{array}{rlrl}
\nu_{\max } & =[u / 2]+[v / 2]+[w / 2], & \mu_{\max } & =[u / 2]+[v / 2] \\
n & =u \bmod 2+v \bmod 2+w \bmod 2+2 \nu, \quad m & =u \bmod 2+v \bmod 2+2 \mu .
\end{array}
$$

The notation $[z]$ is used here to denote the integer part of $z$. The use of the symbols $\bar{\Lambda}$ and $\Lambda$ in $(14)$ is analogous in a sense to the conventional use of symbols such as $\sum$ and $\Pi$, in that it allows a running index to enumerate expressions of a common form, that are to be considered one by one. The expression $\bigwedge_{i=i_{1}}^{i_{2}} x_{i}$ denotes the list of objects $x_{i_{1}}, x_{i_{1}+1}, \cdots, x_{i_{2}}$. If the $L_{j}, j=j_{1}$ to $j_{2}$, denote lists of objects, then $\bar{\bigwedge}_{j=j_{1}}^{j_{2}} L_{j}$ denotes the list that consists of the objects which form the lists $L_{j_{1}}, L_{j_{1}+1}, \cdots, L_{j_{2}}$, taken in turn.

It would be possible to express the position of the $a_{u, v, w}^{n, m}$ coefficient of given $u, v$, $w, n, m$ values, in the sequence of $a_{u, v, w}^{n, m}$ coefficients that is defined by (12) and (14) as a function of $u, v, w, n$ and $m$. It seemed simpler however to construct an expression for the position of an $a_{u, v, w}^{n, m}$ coefficient in the sequence of coefficients that is defined by (15):

$$
\begin{aligned}
s=0(1) s_{\max }, & u=0(1) s, & v=0(1) s-u, \\
w=s-u-v, & n=0(1) s, & m=0(1) n .
\end{aligned}
$$

This sequence of $a_{u, v, w}^{n, m}$ coefficients can be written, using the $\bar{\Lambda}$ and $\bigwedge$ symbols, as

$$
\bigwedge_{s=0}^{s_{\max }} \bigwedge_{u=0}^{s} \bigwedge_{v=0}^{s-u} \bigwedge_{n=0}^{s} \bigwedge_{m=0}^{n} a_{u, v, w}^{n, m}
$$

The list of $a_{u, v, w}^{n, m}$ coefficients contains zero elements for $u, v, w, n, m$ values that do not satisfy the conditions (3). The position (or index) of an $a_{u, v, w}^{n, m}$ coefficient of given $u, v, w, m, n$ values in this list, however, is given by the expression

$$
\begin{aligned}
\sum_{k=0}^{s-1}\{(k+1)(k+2) / 2\}^{2} & +\left\{v+\sum_{j=0}^{u-1}(s+1-j)\right\} \\
& \times\{(s+1)(s+2)\} / 2+n(n+1) / 2+m+1 .
\end{aligned}
$$

A recurrence scheme that generates the list of $a_{u, v, w}^{n, m}$ coefficients that is defined by (15) and (16) can be mechanized very easily, as a process which by-passes the recurrence formulae when the $u, v, w, n, m$ values make the coefficient zero. This scheme uses the expression (17) to find the coefficients in the growing list that are needed in successive applications of the recurrence formulae. The zero entries can then be deleted by an editing process that forms the more concise list (14). This procedure is used by the author's programs which produced Table 1.

The construction of expressions such as (17) for elaborate combinations of 
limits on the $\bar{\bigwedge}$ and $\bigwedge$ symbols in expressions such as (14) are algebraic problems rather than programming problems, even though they are of prime concern in the design of actual programs.

4. Recurrence Formulae for the $b_{u, v, w}^{n, m}$ Coefficients. The recurrence relationships for the Legendre functions are used to derive recurrence relationships for the $b_{u, v, w}^{n, m}$ coefficients in much the same way as for the $a_{u, v, w}^{n, m}$ coefficients. Thus Equation (4) can be used to construct two series for

$$
P_{n+1}^{m}(\cos \theta) \underset{\sin }{\cos } m \phi .
$$

One series is obtained by writing $n+1$ in place of $n$, on both sides of Equation (4). The other series is obtained by expressing

$$
P_{n+1}^{m}(\cos \theta) \underset{\sin }{\cos } m \phi
$$

in terms of

$$
P_{n}^{m}(\cos \theta) \underset{\sin }{\cos } m \phi
$$

and

$$
P_{n-1}^{m}(\cos \theta) \underset{\sin }{\cos } m \phi
$$

expanding these two latter surface harmonics by Equation (4), writing $(z / r)$ for the factor $\cos \theta$ that multiplies the harmonic of order $n$, and relabelling and rearranging the products of Cartesian coordinates that result. Equating coefficients of $(x / r)^{u}(y / r)^{v}(z / r)^{w}$ gives

$$
b_{u, v, w}^{n+1, m}=\frac{2 n+1}{n-m+1} b_{u, v, w-1}^{n, m}-\frac{n+m}{n-m+1} b_{u, v, w}^{n-1, m} .
$$

The corresponding equation that is obtained by expressing

$$
P_{n+1}^{m+1}(\cos \theta) \underset{\sin }{\cos }\{(m+1) \phi\}
$$

in terms of

$$
P_{n}^{m}(\cos \theta) \underset{\sin }{\cos } m \phi
$$

and

$$
P_{n-1}^{m+1}(\cos \theta) \underset{\sin }{\cos }\{(m+1) \phi\}
$$

is

$$
b_{u, v, w}^{n+1, m+1}=b_{u, v, w}^{n-1, m+1}-(2 n+1) b_{u-1, v, w}^{n, m}+(-1)^{v}(2 n+1) b_{u, v-1, w}^{n, m} .
$$

A recurrence scheme to construct the $b_{u, v, w}^{n, m}$ coefficients can start with the single value

$$
b_{0,0,0}^{0,0}=1 .
$$




\section{Table I}

$a(u, v, w, n, m)$ coefficients

$a(u, v, w, n, m)$ coefficients

\begin{tabular}{|c|c|c|c|c|c|}
\hline & $\mathbf{v}$ & $\mathbf{w}$ & $\mathbf{n}$ & m & $\mathbf{a}$ \\
\hline 0 & 0 & 0 & 0 & 0 & $1 / 1$ \\
\hline 0 & 0 & 1 & 1 & 0 & $1 / 1$ \\
\hline 0 & 1 & 0 & 1 & 1 & $-1 / 1$ \\
\hline 1 & 0 & 0 & 1 & 1 & $-1 / 1$ \\
\hline 0 & 0 & 2 & $\begin{array}{l}0 \\
2\end{array}$ & $\begin{array}{l}0 \\
0\end{array}$ & $\begin{array}{l}1 / 3 \\
2 / 3\end{array}$ \\
\hline $\mathbf{0}$ & 1 & 1 & 2 & 1 & $-1 / 3$ \\
\hline 0 & 2 & 0 & $\begin{array}{l}0 \\
2 \\
2\end{array}$ & $\begin{array}{l}0 \\
0 \\
2\end{array}$ & $\begin{array}{r}1 / 3 \\
-1 / 3 \\
-1 / 6\end{array}$ \\
\hline 1 & 0 & 1 & 2 & 1 & $-1 / 3$ \\
\hline 1 & 1 & 0 & 2 & 2 & $1 / 6$ \\
\hline 2 & 0 & 0 & $\begin{array}{l}0 \\
2 \\
2\end{array}$ & $\begin{array}{l}0 \\
0 \\
2\end{array}$ & $\begin{array}{r}1 / 3 \\
-1 / 3 \\
1 / 6\end{array}$ \\
\hline 0 & 0 & 3 & $\begin{array}{l}1 \\
3\end{array}$ & $\begin{array}{l}0 \\
0\end{array}$ & $\begin{array}{l}3 / 5 \\
2 / 5\end{array}$ \\
\hline 0 & 1 & 2 & $\begin{array}{l}1 \\
3\end{array}$ & $\begin{array}{l}1 \\
1\end{array}$ & $\begin{array}{l}-1 / 5 \\
-2 / 15\end{array}$ \\
\hline 0 & $\mathcal{2}$ & 1 & $\begin{array}{l}1 \\
3 \\
3\end{array}$ & $\begin{array}{l}0 \\
0 \\
2\end{array}$ & $\begin{array}{l}1 / 5 \\
-1 / 5 \\
-1 / 30\end{array}$ \\
\hline 0 & 3 & 0 & $\begin{array}{l}1 \\
3 \\
3\end{array}$ & $\begin{array}{l}1 \\
1 \\
3\end{array}$ & $\begin{array}{c}-3 / 5 \\
1 / 10 \\
1 / 60\end{array}$ \\
\hline 1 & 0 & $\mathcal{2}$ & $\begin{array}{l}1 \\
3\end{array}$ & $\begin{array}{l}1 \\
1\end{array}$ & $\begin{array}{l}-1 / 5 \\
-2 / 15\end{array}$ \\
\hline 1 & 1 & 1 & 3 & 2 & $1 / 30$ \\
\hline 1 & 2 & 0 & $\begin{array}{l}1 \\
3 \\
3\end{array}$ & $\begin{array}{l}1 \\
1 \\
3\end{array}$ & $\begin{array}{c}-1 / 5 \\
1 / 30 \\
1 / 60\end{array}$ \\
\hline 2 & 0 & 1 & $\begin{array}{l}\mathbf{1} \\
\mathbf{3} \\
\mathbf{3}\end{array}$ & $\begin{array}{l}0 \\
0 \\
2\end{array}$ & $\begin{array}{c}1 / 5 \\
-1 / 5 \\
1 / 30\end{array}$ \\
\hline 2 & 1 & 0 & $\begin{array}{l}1 \\
3 \\
3\end{array}$ & $\begin{array}{l}1 \\
1 \\
3\end{array}$ & $\begin{array}{c}-1 / 5 \\
1 / 30 \\
-1 / 60\end{array}$ \\
\hline & 0 & 0 & $\begin{array}{l}1 \\
3 \\
3\end{array}$ & $\begin{array}{l}1 \\
1 \\
3\end{array}$ & $\begin{array}{c}-3 / 5 \\
1 / 10 \\
-1 / 60\end{array}$ \\
\hline
\end{tabular}

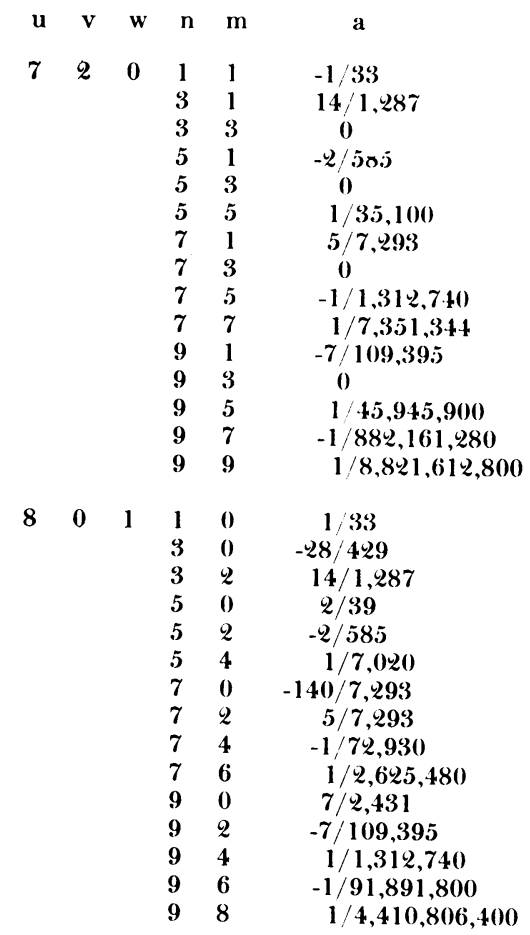

$\begin{array}{llllll}8 & 1 & 0 & 1 & 1 & -1 / 33\end{array}$

$14 / 1,287$

$-7 / 1,287$

$-2 / 585$

$1 / 2,340$

$-1 / 14,040$

$5 / 7,293$

$-1 / 24,310$

$1 / 525,096$

$-1 / 5,250,960$

$-7 / 109,395$

$1 / 437,580$

$-1 / 18,378,360$

$1 / 630,115,200$

$-1 / 8,821,612,800$

$\begin{array}{llllll}9 & 0 & 0 & 1 & 1 & -3 / 11\end{array}$

$14 / 143$

$-7 / 429$

$-2 / 65$

$1 / 780$

$-1 / 7,800$

$15 / 2,431$

$-3 / 24,310$

$1 / 291,720$

$-1 / 4,084,080$

$-7 / 12,155$

$1 / 145,860$

$-1 / 10,210,200$

$1 / 490,089,600$

$-1 / 8,821,612,800$ 
Table 2

$b(n, m, u, v, w)$ coefficients

$b(n, m, u, v, w)$ coefficients

\begin{tabular}{|c|c|c|c|c|c|}
\hline & m & \multicolumn{2}{|c|}{$\begin{array}{ll}\mathbf{u} & \mathbf{v}\end{array}$} & w & b \\
\hline () & o & 0 & 0 & 0 & $1 / 1$ \\
\hline 1 & 0 & 0 & 0 & 1 & $1 / 1$ \\
\hline \multirow[t]{2}{*}{1} & 1 & 0 & 1 & 0 & $-1 / 1$ \\
\hline & & 1 & 0 & 0 & $-1 / 1$ \\
\hline \multirow[t]{2}{*}{$z$} & 0 & o & 0 & 0 & $-1 / 2$ \\
\hline & & 0 & 0 & $\mathcal{2}$ & $3 / 2$ \\
\hline \multirow[t]{2}{*}{$\mathcal{2}$} & 1 & 0 & 1 & 1 & $-3 / 1$ \\
\hline & & 1 & 0 & 1 & $-3 / 1$ \\
\hline \multirow[t]{3}{*}{$z$} & 2 & 0 & 2 & 0 & $-3 / 1$ \\
\hline & & 1 & 1 & 0 & $6 / 1$ \\
\hline & & 2 & 0 & 0 & $3 / 1$ \\
\hline \multirow[t]{2}{*}{3} & 0 & 0 & 0 & 1 & $-.3 / 2$ \\
\hline & & 0 & 0 & 3 & $5 / 2$ \\
\hline \multirow[t]{4}{*}{3} & 1 & 0 & 1 & 0 & $3 / 2$ \\
\hline & & 0 & 1 & $\mathcal{z}$ & $-15 / \mathcal{Q}$ \\
\hline & & 1 & 0 & 0 & $3 / 2$ \\
\hline & & 1 & 0 & $\mathcal{2}$ & $-15 / 2$ \\
\hline \multirow[t]{3}{*}{3} & 2 & 0 & 2 & 1 & $-15 / 1$ \\
\hline & & 1 & 1 & 1 & $30 / 1$ \\
\hline & & 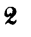 & 0 & 1 & $15 / 1$ \\
\hline \multirow[t]{4}{*}{3} & 3 & 0 & 3 & 0 & $15 / 1$ \\
\hline & & 1 & 2 & 0 & $45 / 1$ \\
\hline & & 2 & 1 & 0 & $-45 / 1$ \\
\hline & & 3 & 0 & 0 & $-15 / 1$ \\
\hline \multirow[t]{3}{*}{4} & 0 & 0 & 0 & 0 & $3 / 8$ \\
\hline & & 0 & 0 & 2 & $-15 / 4$ \\
\hline & & 0 & 0 & 4 & $35 / 8$ \\
\hline \multirow[t]{4}{*}{4} & 1 & 0 & 1 & 1 & $15 / 2$ \\
\hline & & 0 & 1 & 3 & $-35 / 2$ \\
\hline & & 1 & 0 & 1 & $15 / 2$ \\
\hline & & 1 & 0 & 3 & $-35 / 2$ \\
\hline \multirow[t]{6}{*}{4} & $\boldsymbol{2}$ & $\mathbf{0}$ & $\boldsymbol{z}$ & 0 & $15 / 2$ \\
\hline & & 0 & 2 & 2 & $-105 / 2$ \\
\hline & & 1 & 1 & 0 & $-15 / 1$ \\
\hline & & 1 & 1 & $\mathcal{2}$ & $105 / 1$ \\
\hline & & 2 & 0 & 0 & $-15 / 2$ \\
\hline & & 2 & 0 & 2 & $105 / 2$ \\
\hline \multirow[t]{4}{*}{4} & 3 & 0 & 3 & 1 & $105 / 1$ \\
\hline & & 1 & 2 & 1 & $315 / 1$ \\
\hline & & 2 & 1 & 1 & $-315 / 1$ \\
\hline & & 3 & 0 & 1 & $-105 / 1$ \\
\hline \multirow[t]{5}{*}{4} & 4 & 0 & 4 & 0 & $105 / 1$ \\
\hline & & 1 & 3 & 0 & $-420 / 1$ \\
\hline & & $\boldsymbol{z}$ & 2 & 0 & $-630 / 1$ \\
\hline & & 3 & 1 & 0 & $420 / 1$ \\
\hline & & 4 & 0 & 0 & $105 / 1$ \\
\hline
\end{tabular}

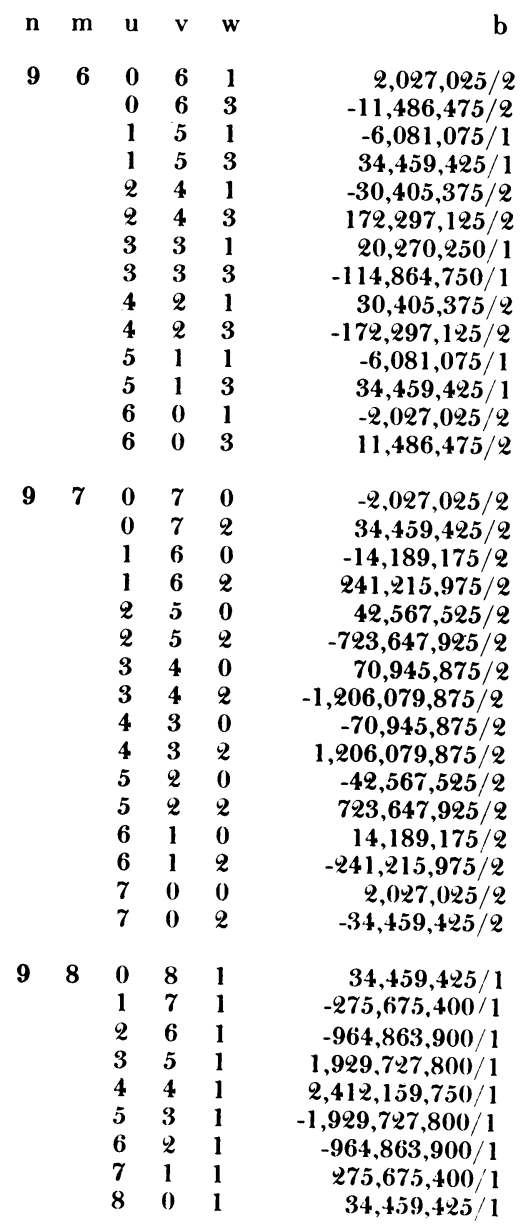

$\begin{array}{lllll}9 & 9 & 0 & 9 & 0\end{array}$

$\begin{array}{lll}1 & 8 & 0 \\ 2 & 7 & 0 \\ 3 & 6 & 0 \\ 4 & 5 & 0 \\ 5 & 4 & 0 \\ 6 & 3 & 0 \\ 7 & 2 & 0 \\ 8 & 1 & 0 \\ 9 & 0 & 0\end{array}$

$-34,459,425 / 1$ $-310,134,825 / 1$ $1,240,539,300 / 1$ $2,894,591,700 / 1$ $-4,341,887,550 / 1$ $-4,341,887,550 / 1$ $\mathbf{2 , 8 9 4 , 5 9 1 , 7 0 0 / 1}$ $1,240,539,300 / 1$ $-310,134,825 / 1$ $-34,459,425 / 1$ 
Equation (18) is used when $m=0$ and Equation (19) when $m>0$. The Equations (18)-(20) permit the $b_{u, v, w}^{n, m}$ coefficients to be computed in the order

$$
\begin{array}{rlrl}
n & =0(1) n_{\max } ; & m=0(1) n ; \quad u=0(1) m ; \\
v=m-u ; & w=(n-m) \bmod 2(2)(n-m) .
\end{array}
$$

The indexing is simplified by constructing the sequence of $b_{u, v, w}^{n, m}$ coefficients (22) that contains zero elements, and removing these in a subsequent operation

$$
\bigwedge_{n=0}^{n_{\max }} \bigwedge_{m=0}^{n} \bigwedge_{u=0}^{m} \bigwedge_{w=0}^{n-m} b_{u, m-u, w}^{n, m} .
$$

The position of the $b_{u, v, w}^{n, m}$ coefficient of given $n, m, u, v, w$ values in this list is given by

$$
\begin{aligned}
\sum_{k=0}^{n-1}\{(k+1)(k+2)(k+3) / 6\}+\sum_{j=0}^{m-1}\left\{n(j+1)-j^{2}+1\right\} \\
+u(n-m+1)+w+1 .
\end{aligned}
$$

Table 2 was produced in this way by generating a table of the form (22) and then editing out elements that are zero as a consequence of (3).

5. Checking and Printing Procedures. Two methods were used to check the tables that were produced in the manner which has been described in the preceding sections. The first method involved the evaluation of the left- and right-hand sides of Equations (2) and (4), using values of $x, y, z$ for which the circular functions of $\theta$ and $\phi$ are rational numbers.

The second method used the orthogonality of the $a$ 's and $b$ 's to check both tables against each other. If

$$
c\left(n, m, n^{\prime}, m^{\prime}\right)=\sum_{u, v, w} b_{u, v, w}^{n, m} a_{u, v, w}^{n^{\prime}, m^{\prime}}
$$

where the summation is taken over the $u, v, w$ values that are allowed by (5), and $v$ is always even or always odd, then

$$
c\left(n, m, n^{\prime}, m^{\prime}\right)=\delta_{n, n^{\prime}} \delta_{m, m^{\prime}} .
$$

A check program was written to read the output decks of the programs which generated the $a_{u, v, w}^{n, m}$ and $b_{u, v, w}^{n, m}$ coefficients, and to form the quantities $c\left(n, m, n^{\prime}, m^{\prime}\right)$. These were printed and checked visually.

The tables of $a_{u, v, w}^{n, m}$ and $b_{u, v, w}^{n, m}$ on punched cards were used as input to the TABPRINT program [5]. This produced a punched paper tape that was then used to control the operations of a Photon S-560 System, to typeset the tables photographically. The printed tables were thus produced from the checked computer output without the need for manual keyboard action.

Acknowledgments. This work formed part of a program of research that is supported by the U. S. Army, Navy and Air Force and by the National Science Foundation.

Cooperative Computing Laboratory

Massachusetts Institute of Technology

Cambridge, Massachusetts 
1. E. W. Hobson, The Theory of Spherical and Ellipsoidal Harmonics, Cambridge, 1931.

2. M. P. BARNETT, "The evaluation of molecular integrals by the zeta function method," Methods in Computational Physics, Vol. II, p. 95, Academic Press, New York, 1963.

3. A. B. Otis, Jr. \& M. P. Barnetr, Surface Harmonic Expansions of Products of Cartesian Coordinates, Technical Note No. 26, Cooperative Computing Laboratory, Massachusetts Institute of Technology, Cambridge, Mass., 1963.

4. M. P. Barnett, "Indexing and the $\Lambda$ notation," Comm. $A C M$, v. 6, 1963, p. 740.

5. M. P. BarnetT, D. A. LuCE \& D. J. Moss, "Computer generation of photocomposing control tapes. II," Amer. Documentation, v. 15, 1964, p. 115. 